\title{
ACTIVIDAD ANTIBACTERIAL Y COMPOSICIÓN QUIIMICA DEL ACEITE ESENCIAL DE LA Aloysia aloysioides Loes
}

\author{
Carmen Huanca Miranda ${ }^{1}$, Nino Castro Mandujano ${ }^{1 *}$, Jesús López Rodilla², \\ Nelson Bautista Cruz ${ }^{3}$
}

\begin{abstract}
RESUMEN
En la presente investigación se estudió al aceite esencial de la "lima-lima" (Aloysia aloysioides Loes y Moldenke), fue colectada en el distrito de Tintay, Apurímac-Perú, los pobladores de esta zona, lo utilizan por sus propiedades antiinflamatorias en forma de emplastos. El objetivo es extraer y caracterizar al aceite esencial de la Aloysia aloysioides Loes \& Moldenke y evaluar la actividad antibacteriana. A partir de 674 gramos de muestra fresca (ramas) se obtuvo el aceite esencial por destilación por arrastre con vapor, luego, se caracterizó realizando un análisis fisicoquímico, cromatográfico por CG-EM, espectroscópico FT-IR y el análisis antibacteriano. Se obtuvo el aceite esencial con un rendimiento de $0,38 \%$; se identificó al $\beta$-Cariofileno (10,88\%), limonelo (9.9\%), p-cimeno (7,22\%), Oxido de cariofileno (5,98 \%), Nerolidol (4,96 \%) y $\alpha$-humuleno (4,92\%) como componentes mayoritarios. Además, este aceite esencial tiene actividad antibacteriana frente a Staphylococcus aureus, hasta un $73 \%$ de efectividad cercano a la gentamicina; por estas razones podría ser usado en preparaciones farmacéuticas.
\end{abstract}

Palabras claves: Aloysia aloysioides Loes \& Moldenke, actividad antibacteriana, CG-EM.

\section{ANTIBACTERIAL ACTIVITY AND CHEMICAL COMPOSITION OF THE ESSENTIAL OIL OF Aloysia aloysioides Loes}

\begin{abstract}
In the present investigation the essential oil of the "lime-lime" (Aloysia aloysioides Loes and Moldenke) was studied, it was collected in the district of Tintay, Apurímac-Peru, the inhabitants of this area, use it for its anti-inflammatory properties in the form of plasters. The objective is to extract and characterize the essential oil of Aloysia aloysioides Loes \& Moldenke and evaluate the antibacterial activity. From 674 grams of fresh sample (branches) the essential oil was obtained by steam distillation, then it was characterized by performing a physicochemical analysis, chromatographic by GC-MS, spectroscopic FT-IR and antibacterial

\footnotetext{
${ }^{1}$ Laboratorio de Productos Naturales, Departamento de Química Orgánica, Escuela de Química, Facultad de Química e Ingeniería de Química, UNMSM. ocastrom@unmsm.edu.pe.

${ }^{2}$ Departamento de Química, Universidade Beira Interior, Covilhã, Portugal.

${ }^{3}$ Laboratorio de Microbiología, Facultad de Farmacia y Bioquímica - UNMSM
} 
analysis. The essential oil was obtained with a yield of $0.38 \%$; $\beta$-Caryophyllene $(10.88 \%)$, limonel $(9.9 \%)$, p-cymene $(7.22 \%)$, caryophyllene oxide $(5.98 \%)$, Nerolidol $(4.96 \%)$ and $\alpha$-humulene were identified $(4.92 \%)$ as majority components. In addition, this essential oil has antibacterial activity against Staphylococcus aureus, up to a $73 \%$ effectiveness close to gentamicin; for these reasons it could be used in pharmaceutical preparations.

Keywords: Aloysia aloysioides Loes \& Moldenke, antibacterial activity, GC-MS.

\section{INTRODUCCIÓN}

El estudio de varias especies de La familia Verbenaceae han permitido evidenciar su utilidad en la medicina tradicional en malestares del aparato digestivo, aparato reproductor femenino, sistema músculo esquelético y nosologías dependientes de la cultural como antioxidantes, esto debido a compuestos fenólicos contenidos en especies como Aloysia citriodora Palau, esta especie contiene un alto porcentaje de fenólico y estos componentes, generalmente poseen una mayor actividad antioxidante ${ }^{2}$, por otro lado, se ha logrado identificar mediante cromatogramas provenientes de la infusión de las hojas un perfil de polifenoles caracterizados por la presencia de ácido cafeico y flavonoides ${ }^{3}$, los extractos alcohólicos y acuosos poseen actividad antiinflamatoria, atribuida a la presencia de theviridosido, un glucósido iridoide, además, otro componente común en la familia VERBENACEAE, la actividad analgésica se debe a la presencia de verbascósido y en cuanto a la actividad antipirética, el extracto acuoso es ligeramente superior al del paracetamol ${ }^{4}$.

Como agentes antimicrobianos y antimicóticos, los aceites esenciales y extractos de plantas han formado las bases de muchas aplicaciones, que van desde la preservación, procesados de carne hasta productos farmacéuticos, es así como el aceite esencial de tomillo no solo actúa como agente antibacteriano si no también minimiza la oxidación de lípidos además de mejorar sus características sensoriales incluso mejorando la salubridad del producto hasta por dos semanas en almacenamiento refrigerado ${ }^{5}$, además, otros estudios demuestran la amplia actividad antimicrobiana de especies de la familia VERBENÁCEA, como es el caso del aceite esencial de la Aloysia triphylla mostrando efectos sobre el trypanosoma cruzi siendo un tratamiento alternativo frente a los escasos fármacos para su tratamiento ${ }^{6}$. Estudios realizados en Venezuela muestran el amplio espectro antibacteriano contra patógenos genitourinarios y hacen notable su uso con bajas dosis ${ }^{6}$ y el aceite de la Aloysia gratissima es un antibacteriano efectivo contra Staphylococcus aureus y M. luteos e incluso mostró actividad antiedematogena con un gran porcentaje de inhibición de la inflamación siendo esto último aplicado de manera tópica ${ }^{7}$ también muestra actividad antimicrobiana contra Escherichia coli y Bacillus subtillis ${ }^{8}$.

En cuanto a la composición de los aceites la identificación de los analitos se apoya en el uso de la cromatografía gases acoplada a espectrometría de masas", la detección en la Aloysia Triphylla da como componentes principales como 1,8-cineol $(12,4 \%)$, geranial $(9,9 \%)$, 6-metil-5-hepten-2-ona $(7,4 \%)$ y neral $(6,9 \%)$ analizado por CG-EM ${ }^{10}$, por otro lado, para la Aloysia gratissima se logró identificar también metabolitos secundarios de la clase de 
terpenos, un total de 14 componentes y siendo los principales componentes identificados isopinocamphone (trans -3-pinanona) $(25.4 \%)$, limoneno $(15.1 \%)$ y guaiol $(12.7 \%)^{11}$.

Finalmente, la presente investigación tiene por objetivo, extraer y caracterizar al aceite esencial de la Aloysia aloysioides Loes \& Moldenke y evaluar la actividad antibacteriana; además, este estudio será un aporte a la flora peruana ya que no hay ningún estudio fitoquímico de esta planta.

\section{PARTE EXPERIMENTAL}

Material vegetal. La muestra en estudio de Aloysia aloysioides Loes \& Moldenke (ver figura 1), fueron recolectadas en junio de 2019 en el distrito de Tintay provincia de Aymaraes, Abancay (Perú).

Identificación de la muestra. La identificación botánica se realizó en el Museo de Historia Natural de la Universidad Nacional Mayor de San Marcos, por el Mg. Asunción Cano Echevarría, quien nos proporcionó el nombre científico como Aloysia aloysioides Loes \& Moldenke (constancia N 124-USM-2019).

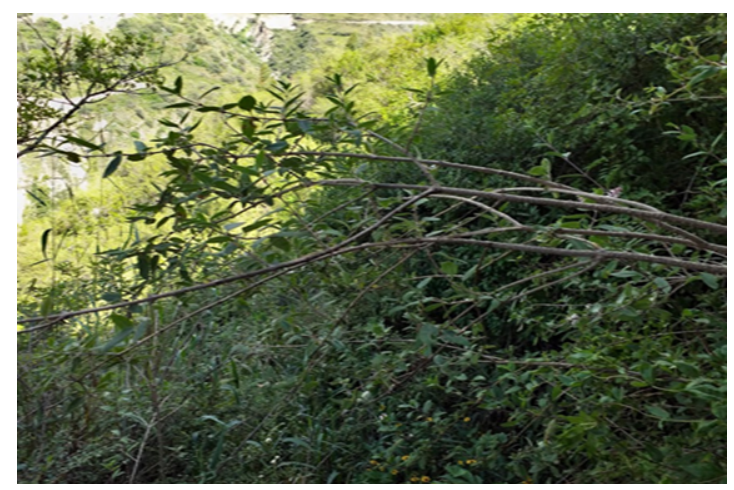

Figura 1. Imagen de la planta en estudio "lima-Lima". 
Tabla 1. Taxonomía de la "lima-lima" Aloysia aloysioides Loes \& Moldenke.

\begin{tabular}{cc}
\hline CARACTERÍSTICA & CLASIFICACIÓN \\
\hline División & Magnoliophyta \\
Clase & Magnoliopsida \\
Subclase & Asteridae \\
Orden & Lamiales \\
Familia & Verbenaceae \\
Genero & Aloysia \\
Especie & Aloysia aloysioides Loes \& Moldenke \\
Nombre vulgar & "lima-lima" \\
\hline
\end{tabular}

Extracción del aceite esencial. La muestra fue sometida a una destilación, por el método de arrastre de vapor de agua, usando 673 gramos, durante 2 horas, usando un equipo semiindustrial de acero inoxidable de 20 litros de capacidad. El aceite esencial obtenido se secó con sulfato de sodio anhidro.

Cromatografía de gases (CG). fue realizado con un cromatógrafo de Agilent ${ }^{\circledR}$ Technologies 7890A GC-System equipado con una columna capilar DB5-MS, 30mx0.25 nm i. d., con film de Polidimetil Siloxane (J \&amp; W LTM Column module) de un espesor de 0,25 $\mu \mathrm{m}$. La temperatura inicial de la columna es de $60^{\circ} \mathrm{C}$ durante 5 minutos, aumentando de $60^{\circ} \mathrm{C}$ a $200^{\circ} \mathrm{C}$, con una rampa de aumento de temperatura de $4^{\circ} \mathrm{C} / \mathrm{min}$, en la siguiente rampa de temperatura aumenta de $200^{\circ} \mathrm{C}$ hasta $\operatorname{los} 280^{\circ} \mathrm{C}$ con una rampa de crecimiento de $10^{\circ} \mathrm{C} / \mathrm{min}$; la temperatura final se mantiene constante durante 35 minutos. La temperatura del inyector es de $250^{\circ} \mathrm{C}$, el detector se encuentra a una temperatura de $180^{\circ} \mathrm{C}$. El gas de arrastre es Helio con un caudal de $1 \mathrm{~mL} / \mathrm{min}$, el volumen de inyección de la muestra es de $1 \mu \mathrm{L}$.

Cromatografía de gases acoplada a espectrometría de masas (CG-EM). realizado en un detector acoplado al cromatógrafo, corresponde a un Agilent ${ }^{\circledR}$ Technologies 5975C, Inert XL MSD con detector Triple-Axis, operando en las condiciones indicadas anteriormente, con un voltaje de ionización de $70 \mathrm{eV}$ y con la fuente iónica a una temperatura de $230^{\circ} \mathrm{C}$. A análisis se realiza aproximadamente en $110 \mathrm{~min}$.

Análisis de los resultados. se realizó con el software Chemstation para el análisis de las áreas obtenidas para los varios componentes, la identificación de los espectros de masas de cada pico se realiza con las siguientes bases de datos: Nist Mass Spectral Software, y Wiley. 


\section{Análisis Microbiológico}

Cepas microbianas. Para el ensayo de actividad antimicrobiana se utilizaron 3 cepas bacterianas y una cepa fúngica (hongo). Todas las cepas utilizadas fueron cepas patógenas aisladas de muestras clínicas humanas proporcionadas por el laboratorio de Microbiología de la Facultad de Farmacia y Bioquímica - UNMSM. Las cepas bacterianas fueron Escherichia coli y Pseudomonas aeruginosa (Gram negativas) y Staphylococcus aureus (Gram positivo) y Candida albicans (hongo).

Preparación de inóculos y ensayo de actividad antimicrobiana. La técnica utilizada fue difusión en pozo en agar. Se realizó en el laboratorio de Microbiología de la Facultad de Farmacia y Bioquímica, Universidad Nacional Mayor de San Marcos. Los microorganismos almacenados en agar inclinado se cultivaron en agar tripticasa soya (TSA) paras las bacterias y en agar Sabouraud dextrosa para el hongo, se incubaron a $37^{\circ} \mathrm{C}$ x $24 \mathrm{~h}$. Después de este tiempo se realizó la preparación de las suspensiones microbianas en suero fisiológico a 0,5 de escala de McFarland. De cada suspensión microbiana se tomó $50 \mu \mathrm{L}$ y se mezcló con $20 \mathrm{~mL}$ de agar Mueller Hinton licuado estéril a $45^{\circ} \mathrm{C}$ para las bacterias y agar Sabouraud para Candida albicans, luego se pasó a placas Petri estéril, se dejó solidificar y se realizó 3 pozos de $6 \mathrm{~mm}$ de diámetro en cada placa utilizando un sacabocados estéril. En un pozo de las placas se colocó $100 \mu \mathrm{g}$ de la muestra (crema), en el segundo pozo se colocó $50 \mu \mathrm{L}$ ( 25 $\mu \mathrm{g} / \mathrm{mL}$ ) de solución gentamicina (control + ) para las bacterias y fluconazol (control + ) para Candida albicans y en el tercer pozo se colocó $50 \mu \mathrm{L}$ de suero fisiológico (control -). Las placas de fueron incubadas a $37^{\circ} \mathrm{C}$ entre 24 a $48 \mathrm{~h}$ en este tiempo se verificó la presencia de los halos de inhibición alrededor de los pozos. En las placas con presencia de halos de inhibición se midieron el diámetro de los halos en milímetros $(\mathrm{mm})$. Los ensayos para la muestra se realizaron por duplicado ${ }^{12,13}$.

\section{RESULTADOS Y DISCUSION}

Se obtuvo un aceite de olor cítrico con leve color amarillo, con un rendimiento de $0,38 \%$, respecto al material muestra inicial, la índice refracción del aceite esencial fue 1,477 y la densidad promedio fue de $0,8525 \mathrm{~g} / \mathrm{ml}$.

\section{Análisis espectroscópico}

\section{Espectroscopía infrarroja}

En la figura 2, se muestra el espectro FT-IR del aceite esencial de Aloysia aloysiodes Loes \& Moldenke, que permitirá identificar los grupos funcionales contenidos en los componentes químicos del aceite esencial 


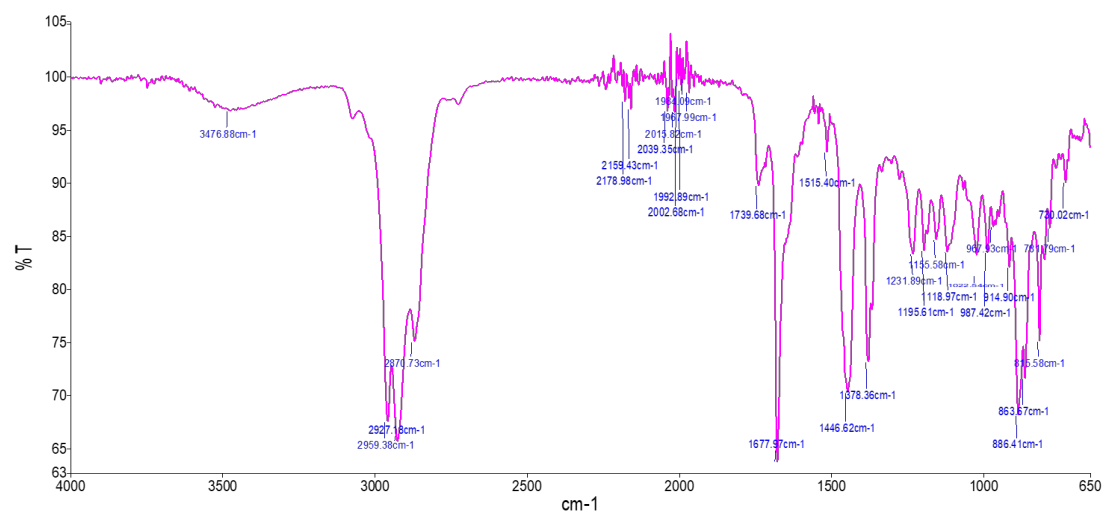

Figura 2. Espectro IR del aceite esencial de Lima-Lima.

En la Tabla $N^{\circ} 1$ se muestran los componentes identificados mediante espectroscopia IR, tales como los dimetilos terminales presentes en el citral, la $\alpha$-tujona y la 6 -metil-5-hepten-2-ona. El estiramiento del enlace $\mathrm{C}=\mathrm{O}$ en 1739 y $1677 \mathrm{~cm}^{-1}$ es característico del grupo carbonilo y evaluado junto con la torsión $\mathrm{C}-\mathrm{H}$ que se aprecia a $1378 \mathrm{~cm}^{-1}$, indican la presencia de aldehídos, posiblemente con enlace $\mathrm{C}=\mathrm{C}$ en su cadena principal debido al pico en $1677 \mathrm{~cm}^{-1}$; esto coincide con la estructura del citral, este compuesto está presente en el aceite esencial de cedrón. Además, se pueden observar picos de absorción a $1446 \mathrm{~cm}^{-1}$, característicos de torsión de tijeras de radicales metilenos (-CH2-) de compuestos cíclicos pequeños $\left(\mathrm{C}_{5}-\mathrm{C}_{8}\right)$. Así mismo, hay picos de absorción en 2959 y $1446 \mathrm{~cm}^{-1}$, que corresponden a grupos metileno terminales $(=\mathrm{CH} 2)$, como los hallados en la molécula del limoneno o el espatulenol. Los picos de absorción en 1195 y $1118 \mathrm{~cm}^{-1}$ son indicio de la presencia de alcoholes secundarios y terciarios debido al estiramiento del enlace $\mathrm{C}-\mathrm{OH}^{14}$. Además, se observa que alrededor de $2000 \mathrm{~cm}^{-1}$, hay varios picos pequeños, el cual nos indica que el aceite esencial tiene aromáticos. 
Tabla 2. Bandas de absorción del espectro infrarrojo del aceite esencial de "Lima-Lima".

\begin{tabular}{|c|c|c|}
\hline $\begin{array}{c}\text { Bandas } \\
\mathbf{c m}^{-1}\end{array}$ & $\begin{array}{c}\text { Grupo } \\
\text { funcional }\end{array}$ & Observaciones \\
\hline 3476 & $\mathrm{O}-\mathrm{H}$ & Una banda de tensión O-H enlazado a un aromático \\
\hline 2959 & $-\mathrm{CH}_{3}$ & $\begin{array}{l}\text { Doblete debido a las vibraciones de tensión asimétricaysimétrica } \\
\text { del C-H independiente de la molécula }\end{array}$ \\
\hline 2959-2870 & $-\mathrm{CH}_{3},-\mathrm{CH}_{2}-$ & $\begin{array}{l}\text { Vibración de alargamiento de enlaces } \mathrm{C} \text { - } \mathrm{H} \text { característico de } \\
\text { compuestos orgánicos. Valor es útil para determinar la hibridación } \\
\text { del átomo de carbono, corresponde a carbono } \mathrm{sp}^{3} \text { (alcano o } \\
\text { posibles grupos alquilo) }\end{array}$ \\
\hline 2870 & $-\mathrm{CH}_{2}-$ & $\begin{array}{l}\text { Vibraciones simétricas del átomo de hidrógeno,independiente } \\
\text { del tamaño de la molécula. }\end{array}$ \\
\hline 1738 & $(\mathrm{C}=\mathrm{O})$ & $\begin{array}{l}\text { Vibración de alargamiento del carbonilo }(\mathrm{C}=\mathrm{O}) \text { valor } \\
\text { característico de aldehídos y cetonas. }\end{array}$ \\
\hline 1446 & $\mathrm{CH}_{2-}$ & Flexión de carbono - hidrógeno. \\
\hline 1446 & $-\mathrm{CH}_{3}$ & Característico del grupo metilo. \\
\hline 1378 & $-\mathrm{CH}_{3},-\mathrm{CH}_{2}-$ & $\begin{array}{l}\text { Característico del isopropil (dos grupos metilo en el mismo } \\
\text { carbono) }\end{array}$ \\
\hline 1364 & $-\mathrm{CH}_{3}$ & Alcano, metilo - geminal. \\
\hline 987 & Alifáticos & Bandas muy débiles con difícil designación \\
\hline $914-967$ & Alifáticos & Bandas muy débiles con difícil designación \\
\hline $815-840$ & $=\mathrm{C}-\mathrm{H}$ & Alquenos, hidrocarburos aromáticos. \\
\hline $730-781$ & $\mathrm{C}-\mathrm{H}$ & Debido a vibraciones del carbono - hidrógeno \\
\hline
\end{tabular}

La identificación de los componentes presentes en el aceite esencial mediante CG-EM, se realizó comparando los tiempos de retención y los espectros de masas mediante búsqueda computarizada en la librería con las siguientes bases de datos: Nist Mass Spectral Software, y Wiley. En la figura 3, tenemos el cromatograma del aceite esencial. 


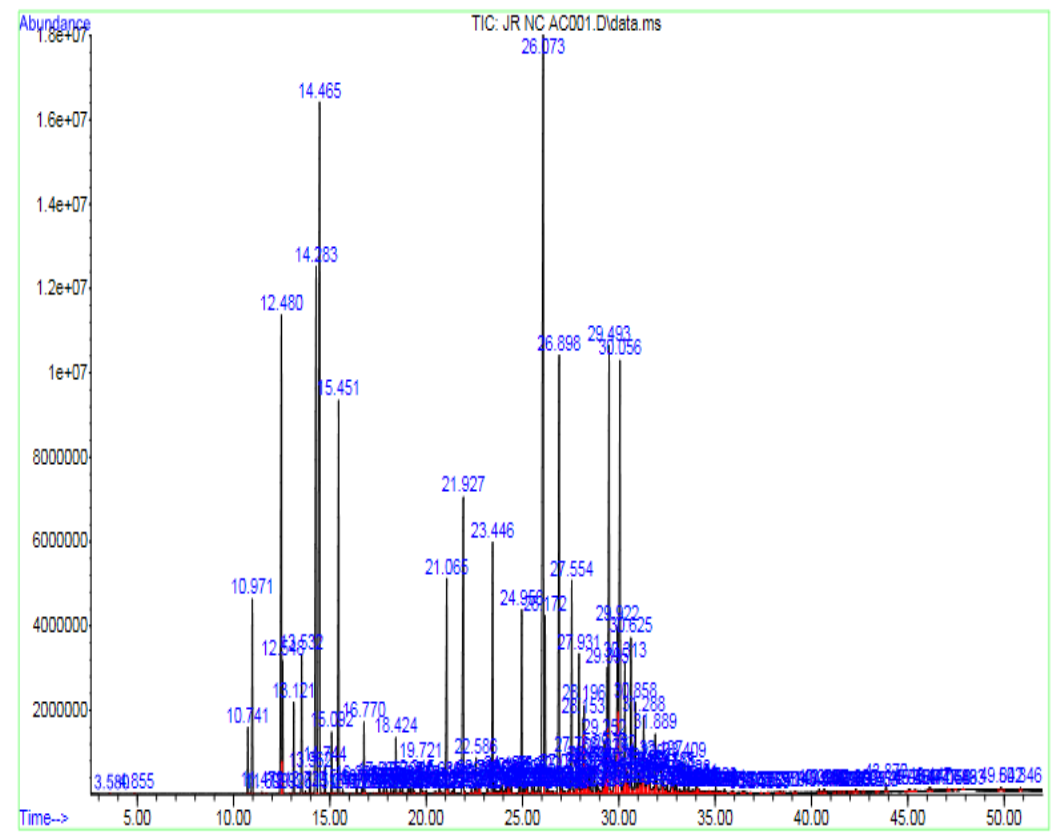

Figura 3. Cromatograma CG_EM del aceite esencial de la Aloysia aloysioides.

Respecto de composición, de los treinta componentes identificados, el 30,81\% monoterpenos, monoterpenos oxigenados de $11,32 \%$, sesquiterpenos $22,94 \%$ y finalmente con un $18,35 \%$ de sesquiterpenos oxigenados estos mostrados en la figura 3. Los componentes mayoritarios identificados fueron: $\beta$-Cariofileno (10,88 \%), limonelo (9,9\%), p-cimeno (7,22\%), Oxido de cariofileno (5,98 \%), Nerolidol (4,96 \%) y $\alpha$-humuleno (4,92\%). Este aceite guarda estrecha relación con el aceite esencial De Aloysia thyphylla siendo esta de la misma familia estudiada en Argentina el $\beta$-Cariofileno (3,3\%) y otra en Venezuela, $\beta$-Cariofileno (2,1\%). 
Tabla 3. La composición del aceite esencial de Lima-Lima.

\begin{tabular}{lccc}
\hline Componente & $\begin{array}{c}\text { Abundancia } \\
\text { \% }\end{array}$ & RT & $\begin{array}{c}\text { Fórmula } \\
\text { global }\end{array}$ \\
\hline ק-Cariofileno & 10,88 & 26,07 & $\mathrm{C}_{15} \mathrm{H}_{24}$ \\
limonelo & 9,49 & 14,46 & $\mathrm{C}_{10} \mathrm{H}_{16}$ \\
p-cimeno & 7,22 & 14,28 & $\mathrm{C}_{10} \mathrm{H}_{14}$ \\
Oxido de cariofileno & 5,98 & 30,06 & $\mathrm{C}_{15} \mathrm{H}_{24} \mathrm{O}$ \\
Sabineno & 5,61 & 12,48 & $\mathrm{C}_{10} \mathrm{H}_{16}$ \\
Nerolidol & 4,96 & 29,49 & $\mathrm{C}_{15} \mathrm{H}_{26} \mathrm{O}$ \\
$\alpha$-humuleno & 4,92 & 26,9 & $\mathrm{C}_{15} \mathrm{H}_{24}$ \\
Y-terpineno & 3,8 & 15,45 & $\mathrm{C}_{10} \mathrm{H}_{16}$ \\
Citral & 3,67 & 21,93 & $\mathrm{C}_{10} \mathrm{H}_{16} \mathrm{O}$ \\
Gemacreno D & 2,22 & 27,33 & $\mathrm{C}_{15} \mathrm{H}_{24}$ \\
Espatulenol & 2,14 & 29,92 & $\mathrm{C}_{15} \mathrm{H}_{24} \mathrm{O}$ \\
neral & 2,29 & 21,06 & $\mathrm{C}_{10} \mathrm{H}_{16} \mathrm{O}$ \\
Acetato de Mirtenilo & 2,31 & 23,45 & $\mathrm{C}_{12} \mathrm{H}_{18} \mathrm{O}_{2}$ \\
$\alpha$-pineno & 1,72 & 10,97 & $\mathrm{C}_{10} \mathrm{H}_{16}$ \\
$\alpha$-felandreno & 1,18 & 13,53 & $\mathrm{C}_{10} \mathrm{H}_{16}$ \\
$\beta$-pineno & 1,05 & 12,55 & $\mathrm{C}_{10} \mathrm{H}_{16}$ \\
Acetato de Geranilo & 1,62 & 24,96 & $\mathrm{C}_{12} \mathrm{H}_{20} \mathrm{O}_{2}$ \\
$\alpha$-Chamigreno & 1,72 & 26,1 & $\mathrm{C}_{15} \mathrm{H}_{24}$ \\
Biciclogermacreno & 1,31 & 27,55 & $\mathrm{C}_{15} \mathrm{H}_{24}$ \\
Germacreno B & 1,2 & 29,39 & $\mathrm{C}_{15} \mathrm{H}_{24}$ \\
Guaiol & 1,24 & 30,18 & $\mathrm{C}_{15} \mathrm{H}_{26} \mathrm{O}$ \\
Oxido Humuleno & 1,59 & 30,37 & $\mathrm{C}_{15} \mathrm{H}_{24} \mathrm{O}$ \\
Endo-1-bourbananol & 0,94 & 30,51 & $\mathrm{C}_{15} \mathrm{H}_{26} \mathrm{O}$ \\
Cadinol Isomero & 0,83 & 31,29 & $\mathrm{C}_{15} \mathrm{H}_{26} \mathrm{O}$ \\
$\beta$-Mirceno & 0,74 & 13,12 & $\mathrm{C}_{10} \mathrm{H}_{16}$ \\
Hidrato de Sesquisabineno & 0,67 & 29,14 & $\mathrm{C}_{15} \mathrm{H}_{26} \mathrm{O}$ \\
Butirato de Geranilo & 0,82 & 28,11 & $\mathrm{C}_{14} \mathrm{H}_{24} \mathrm{O}_{2}$ \\
$\beta$-Bisaboleno & 0,69 & 28,15 & $\mathrm{C}_{15} \mathrm{H}_{24}$ \\
Linelool & 0,61 & 16,77 & $\mathrm{C}_{10} \mathrm{H}_{18} \mathrm{O}$ \\
otros componentes $(>0,5 \%)$ & 17,1 & & \\
TOTAL & 99,9 & & \\
\hline & & & \\
\hline
\end{tabular}

El componente mayoritario es el beta-Cariofileno, con casi $11 \%$ del aceite esencial, además, se verificó la presencia del limoneno, se concluyó que este componente de la planta es el que le proporciona al aceite de lima-lima un sabor y olor agradable, pues su contenido es importante, aunque menor al beta-Cariofileno. Como se puede apreciar la figura 4, el contenido de Sesquiterpenos y Monoterpenos presentes en el aceite de esencial de limalima expresado en porcentajes, cuya diferencia entre ambos componentes es del $7.87 \%$, que hace notable la cantidad elevada de Sesquiterpenos presentes en la Aloysia peruviana y la distingue de otras especies Aloysias reportadas en estudios previos. 


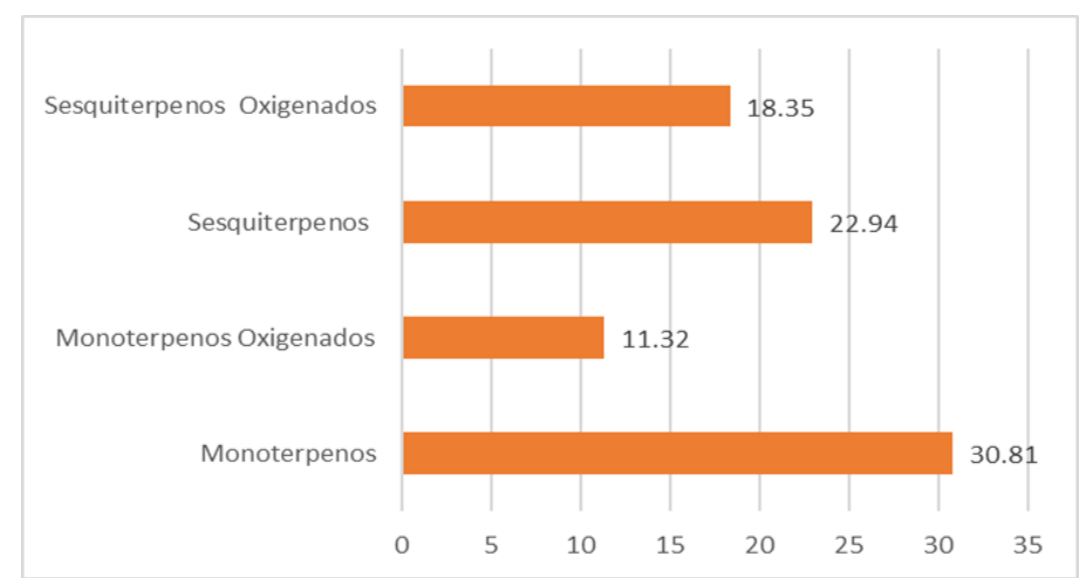

Figura 4. Porcentaje de Terpenos.

Evaluación de la actividad antibacteriana. En la tabla 4 se evidencia que para la muestra existe halo de inhibición solo para Staphylococcus aureus, no existe halos de inhibición para Escherichia coli, Pseudomonas aeruginosa y Candida albicans. Se concluye que el aceite esencial tiene actividad antimicrobiana contra Staphylococcus aureus (Gram positivo) pero no tiene ninguna actividad antimicrobiana contra Escherichia coli y Pseudomonas aeruginosa (Gram negativos) y Candida albicans (hongo). Es preciso indicar que, la muestra fue enfrentada a las cepas clínicas, los cuales son diferentes a las cepas estándar (ATCC). Las cepas clínicas pueden ser mucho más resistentes a comparación de las cepas estándar, pero realizar el ensayo con cepas clínicas se acercan a la realidad si se tiene previsto aplicar el aceite en algún producto.

De acuerdo con los valores obtenidos se observa que el aceite esencial de Aloysia aloysioides Loes \& Moldenke no presentó actividad que otras especies de Aloysia de su misma familia, ya que diversos estudios indican que si son bioactivos frente a Candida albicans, Pseudomonas aeruginosa y Escherichia coli (Demo et. al, 2005 y Duarte et. al, 2007). Esta diferencia se puede atribuir a variación en la composición química del aceite la ausencia del geranial y el porcentaje mínimo de neral el cual en el caso del aceite esencial de Lima -lima no supera el 3\%, también, otro factor a tomar en cuenta es la proporción de Monoterpenos y Sesquiterpenos es muy cercana no mayor al 7,87 \%, finalmente esta composición podría estar influenciada también por las condiciones del medio ambiente donde crece la planta, esto fue demostrado en estudios previos en otras Aloysias. 
Tabla 4. Actividad antibacteriana del aceite esencial de Aloysia aloysioides Loes \& Moldenke contra cepas de referencia.

\begin{tabular}{lllllc}
\hline & \multicolumn{5}{c}{ Diámetro de halos de inhibición (mm) } \\
\cline { 2 - 6 } Microorganismos & $\begin{array}{l}\text { Fluconazol } \\
\text { (control +) }\end{array}$ & $\begin{array}{l}\text { (control +) } \\
\end{array}$ & (Control -) & 1 & $\mathbf{2}$ \\
\hline Escherichia coli & --- & 30 & 0 & 0 & 0 \\
Pseudomonas aeruginosa & --- & 25 & 0 & 0 & 0 \\
Staphylococcus aureus & --- & 26 & 0 & 19 & 19 \\
Candida albicans & 23 & --- & 0 & 0 & 0 \\
\hline
\end{tabular}

\section{CONCLUSIONES}

El aceite esencial de Aloysia aloysioides Loes \& Moldenke, obtenido por el método de destilación por arrastre con vapor de agua, posee un rendimiento RAE\% de 0,38\%. El análisis fisicoquímico del aceite esencial, nos revela que es un líquido de color ligeramente amarillento, olor cítrico herbáceo, sabor ligeramente dulce y cítrico, soluble en alcohol al $96 \%$, el índice de refracción es 1,4798 , la densidad de $0,8561 \mathrm{~g} / \mathrm{mL}$.

Se han identificado y cuantificado 208 compuestos empleando la Cromatografía de Gases acoplada a Espectrometría de Masas (CG-EM) en la muestra de aceite esencial de A. aloysioides Loes \& Moldenke, de estos, 6 son los compuestos mayoritarios, estos son: $\beta$-Cariofileno (10,88\%), limonelo (9,9\%), p-cimeno (7,22\%), Oxido de cariofileno $(5,98 \%)$, Nerolidol (4,96\%) y $\alpha$-humuleno $(4,92 \%)$.

Este aceite esencial es una fuente de sustancias con actividad antibacteriana que podría ser usado en preparaciones farmacéuticas y aplicaciones en la Industria Alimentaria para el tratamiento de infecciones causadas por Staphylococcus aureus. Por otro lado, este es el primer reporte de actividad antibacteriana del aceite esencial de A. aloysioides Loes \& Moldenke contra patógenos.

\section{REFERENCIAS BIBLIOGRÁFICAS}

1. López-Villafranco M, Aguilar-Contreras A, Aguilar-Rodríguez S, Xolalpa-Molina S. Las Verbenaceae empleadas como recurso herbolario en México: una revisión Etnobotánica-Médica. Polibotánica. 2017; 44:195-216.

2. Hashemi S, Mohammad B, Khaneghah A, Koubaa M, Barba F, Abedi E, Niakousari M, Tavakoli J. Extracción de aceite esencial de hojas de Aloysia citriodora Palau usando ultrasonido continuo y pulsado: cinética, actividad antioxidante y antimicrobiana. Process Biochem. 2018; 65:197-204 
3. Wernert M, Wagner M, Gurni A, Carballo M, Ricco R. Estudio de polifenoles de infusiones y cocimientos de hojas de "Cedrón" (Aloysia citrodora Palau) y "Poleo" (Lippia turbinata Griseb.) -Verbenaceae. BLACPMA. 2009; 8(4): 308-311.

4. Seham S, El-Hawary M. Amira Y, Motaal A, Abd-Hameed L. Bioactivities, phenolic compounds and in-vitro propagation of Lippia citriodora Kunth cultivated in Egypt. Bull Fac Pharm Cairo Univ. 2012; 50(1): 1-6.

5. Kassem G, Atta-Alla O, Ali F. Improving the quality of beef burger by adding thyme essential oil and jojoba oil. Arch Zootec. 2011; 60 (231): 787-795.

6. Rojas L, Velasco J, Díaz T, Gil Otaiza R, Carmona J, Usubillaga A. Composición química y efecto antibacteriano del aceite esencial de Aloysia triphylla (L'Hér.) Britton contra patógenos genito-urinarios. BLACPMA. 2010; 9 (1): 56 - 62

7. Vandresen F, Shmitt E, Kato L, Alves De Oliveira C, Bersani A, Da Silva C. Constituintes químicos e avaliação das atividades antibacteriana e antiedematogênica de Aloysia gratissima (Gillies \& Hook.) Tronc. y Aloysia virgata (Ruiz \& Pav.) Pers., Verbenaceae. Rev Bras Farmacogn. 2010; 20(3):317-321.

8. Bailac P, Dellacasa D, Firpo N, Ponzi M. Composición del aceite esencial y actividad antimicrobiana de Aloysia gratissima de San Luis-Argentina. An Asoc Quím Argent. 1999; 87(3):149-153.

9. Stashenko E, Jairo M. Algunos aspectos prácticos para identificación de analitos por cromatografía de gases acoplada a espectrometría de masas. Scientia Chrom. 2010; 2(1):29-47.

10. Bellakhdar J, Idrissi A, Canigueral S, Iglesias J, Vila R. Composition of lemon verbena (Aloysia triphylla (L`Herit) Briton) oil of Morococcan. J Essent Oil Res. 1994; 6(5):523 $-526$.

11. Trovati G, Gilberto O, Chierice, Sanches E, Galhiane M. Essential Oil Composition of Aloysia gratissima From Brazil. J Essent Oil Res. 2010; 4:325-326,

12. Odeniyi MA, Okumah VC, Adebayo-Tayo BC, Odeniyi OA. Green synthesis and cream formulations of silver nanoparticles of Nauclea latifolia (African peach) fruit extracts and evaluation of antimicrobial and antioxidant activities. Sustain Chem Pharm. 2020;15:100197. doi:10.1016/j.scp.2019.100197

13. Torres-Chati J, León-Quispe J, Tomas-Chota G. Actividad antibacteriana y antifúngica de extractos de hojas de Luma chequen (Molina) A. Gray "arrayán" frente a patógenos de origen clínico. Rev Soc Ven Microbiol. 2017; 37(1): 10-16.

14. Pretsch E. Tablas para la elucidación estructural de compuestos orgánicos por métodos espectroscópicos. Madrid: Editorial Alhambra; 2015.

15. Darwish RS, Hammoda HM, Ghareeb DA, Bellah El Naggar EM, Harraz FM, \& Shawky E. Efficacy-directed discrimination of the essential oils of three Juniperus species based on their in-vitro antimicrobial and anti-inflammatory activities. J Ethnopharmacol. 2020; 259:112971. doi: 10.1016/j.jep.2020.112971. 